Bowman, R.E., Maclusky, N.J., Diaz, S., Zrull, M.C., \& Luine, V.N. (2006). Aged rats: Sex differences and responses to chronic stress. Brain Research, 1126(1): 156-166. (December 2006) Published by Elsevier Science Limited (ISSN: 1872-6240). DOI:10.1016/j.brainres.2006.07.047

\title{
Aged rats: Sex differences and responses to chronic stress
}

Rachel E. Bowman, Neil J. Maclusky, Samantha E. Diaz, Mark C. Zrull, and Victoria N. Luine

\begin{abstract}
Cognitive, as well as physiological, sex differences exist in young adult rats under both basal conditions and following chronic stress; however, few studies have examined whether sex differences remain in aged subjects and whether responses to stress are altered. We compared aged male and female Fischer 344 rats (21.5 months at testing) without stress and when given 21 days of restraint for $6 \mathrm{~h}$ /day on locomotion, anxiety-related behaviors, object recognition (non-spatial memory), object placement (spatial memory), body weight and serum steroid hormone levels. Control (unstressed) females had lower levels of estradiol and testosterone and higher corticosterone than males, and stress had no lasting effect on hormone concentrations. Females weighed less than males and showed less weight loss with stress. Locomotion measures on an open field were similar in the sexes and unaffected by stress. Anxiety-related behavior measures on the field showed that males were generally more anxious and that stress increased male, but decreased, female anxiety-related behaviors. In memory testing, exploration of objects was not different between the sexes, with or without stress, while stress increased exploration in both sexes during object recognition trials. Both males and females, regardless of treatment, discriminated between old and new objects at short, but not long, intertrial delays. The typical advantage of young males for spatial memory performance was not observed in aged subjects on the object placement tasks. Stress-dependent enhancements in females and impairments in males for object placement are reported for young rats, but in aged rats, neither sex was altered by stress. Current data suggest that aging is associated with changes in the pattern of sex differences present in young adult rats in some behaviors and in the behavioral responses to stress.
\end{abstract}




\section{INTRODUCTION}

It is generally well accepted that males outperform females on cognitive tasks that require the use of spatial learning and memory. These cognitive sex differences have been observed in humans (Astur et al., 2004; Driscoll et al., 2005; Sanders et al., 1982), non-human primates (Lacreuse et al., 2005) and rodents (Bucci et al., 1995, Gresack and Frick, 2003, Perrot-Sinal et al., 1996, Roof and Havens, 1992 and Williams et al., 1990). Spatial learning and memory is highly dependent on hippocampal functioning (McNaughton et al., 1989 and O'Keefe and Dostrovsy, 1971), and the better performance by males has been shown on a variety of behavioral paradigms that utilize visuo-spatial cues including the Y-maze (Conrad et al., 2003), radial arm maze (Roof, 1993 and Williams et al., 1990), Morris water maze (Roof, 1993 and Kitraki et al., 2004) and the object placement (Beck and Luine, 2002).

Interestingly, sex differences in cognitive function are altered in young adult rats following stress exposure. In general, short periods of stress are adaptive and longer periods of stress can be maladaptive to young adult male rats on both spatial and non-spatial learning and memory paradigms (Beck and Luine, 2002, Conrad et al., 1996, Conrad et al., 2003, Kitraki et al., 2004, Luine et al., 1994, McLay et al., 1998 and Mizoguchi et al., 2000). Young adult female rats are more resistant than males to the impairing effects of stress, demonstrating enhanced performance on the same task/test that males showed cognitive impairment (Bowman et al., 2001, Bowman et al., 2002, Conrad et al., 2003 and Kitraki et al., 2004). For example, following chronic restraint stress ( $6 \mathrm{~h} /$ day, 21 days), males are impaired, but stressed females demonstrate enhanced performance as compared to their control counterparts on the radial arm maze (Bowman et al., 2001), the Y-maze (Conrad et al., 2003), object placement (Beck and Luine, 2002) and the Morris water maze (Kitraki et al., 2004). Importantly, the apparent female resistance to chronic stress effects on both spatial and non-spatial cognitive tasks appears to be influenced by both organizational and activational influences of estrogen (Bowman et al., 2002 and Conrad et al., 2003).

The normal aging process is associated with profound physiologic changes including declines in cognitive functioning and alterations in hypothalamic-pituitary-adrenocortical (HPA) axis activity in response to stressful events. Most work on cognitive alterations in aged animals has focused on the hippocampus, and declines in spatial performance have consistently been reported (Barnes, 1988 and Gallagher and Pelleymounter, 1988). Impairments in spatial memory have been associated with changes in hippocampal morphology and plasticity in aged animals (for a review, see McEwen, 1999, 2001). Age-related changes are also observed in the response to stress experiences by the hypothalamic-pituitary-adrenocortical (HPA) axis. Aged rats generally show higher basal levels of corticosterone and a delay in termination of secretion following stress exposure (Bodnoff et al., 1995).

Central to both cognitive changes and HPA functioning in aged animals is the hippocampus. The hippocampus is involved in various aspects of learning and memory, has the highest density of glucocorticoid (GC) receptors in the brain and is involved in the regulation of the HPA and in behavioral responses to stress. Furthermore, the hippocampus is vulnerable to the aging process and undergoes decreases in neuronal density in response to heightened HPA activity 
during aging (Issa et al., 1990; for a review, see Sapolsky, 1999). Estrogen-induced synaptic plasticity in the hippocampus is also altered during aging, and aged females lack the increased spine density observed in young females in response to estrogen treatment (Adams et al., 2001 and Adams et al., 2002). Thus, the hippocampus provides an ideal model for studying cognitive functioning under both basal conditions as well as the neurobiological effects of increased levels of GC associated with stress.

While age-related changes in rodent cognitive functioning are well documented (Luine and Hearns, 1990, Luine et al., 1990, Nicholson et al., 2004 and Shukitt-Hale et al., 2004), most work has focused on males and little attention has been paid to whether typical sex differences in spatial memory persist in aged animals. There is a surprising lack of research into the effects of chronic stress on behavior and cognitive functioning in aged rodents, particularly with regard to possible sex differences. In summary, under non-stressed conditions, young rodents show several behavioral sex differences such as males showing better performance of spatial memory tasks (Gresack and Frick, 2003, Roof and Havens, 1992 and Williams et al., 1990) and greater anxiety-related behavioral levels (Beck and Luine, 2002, Bowman et al., 2004 and Wilson et al., 2004). In addition, chronic restraint stress elicits many sex-specific responses with regard to brain morphology (Galea et al., 1997), central neurotransmitter levels (Beck and Luine, 2002; for a review, see Bowman et al., 2003) and cognitive functioning (for a review, see Bowman, 2005) in young adult rats. The purpose of the current experiment was to examine whether these sex-dependent behavioral differences and responses to stress persist in aged animals when the hippocampus is vulnerable to insult (McEwen, 2002 and Small et al., 2004), gonadal steroid levels have fallen (Chen et al., 1994, LeFevre and McClintock, 1988 and Luo et al., 2005) and HPA activity is enhanced (Herman et al., 2001).

\section{RESULTS}

\section{Body Weights}

Body weights for all subjects, across the 21-day stress period, are shown in Fig. 1. A significant difference was observed between males and females, with the males weighing more than the females $\left(F_{1,161}=668.35, p<0.001\right)$. Stressed animals weighed less than controls $\left(F_{1,161}=8.06\right.$, $p<0.007$ ) (338.30 versus 360.44 , stress and controls, respectively, data in grams). A main effect of day $\left(F_{1,161}=189.93, p<0.001\right)$ revealed that body weights decreased across the 21 day period. In addition, there was a sex $\times$ day interaction $\left(F_{1,161}=35.75, p<0.001\right)$, a treatment $\times$ stress day interaction $\left(F_{1,161}=58.26, p<0.001\right)$ and a sex $\times$ treatment $\times$ day effect

$\left(F_{1,161}=13.84, p<0.001\right)$, and these results revealed that stressed males lost more weight than control males across the 21-day period, but this weight loss effect was less pronounced in stressed females compared to control females. 


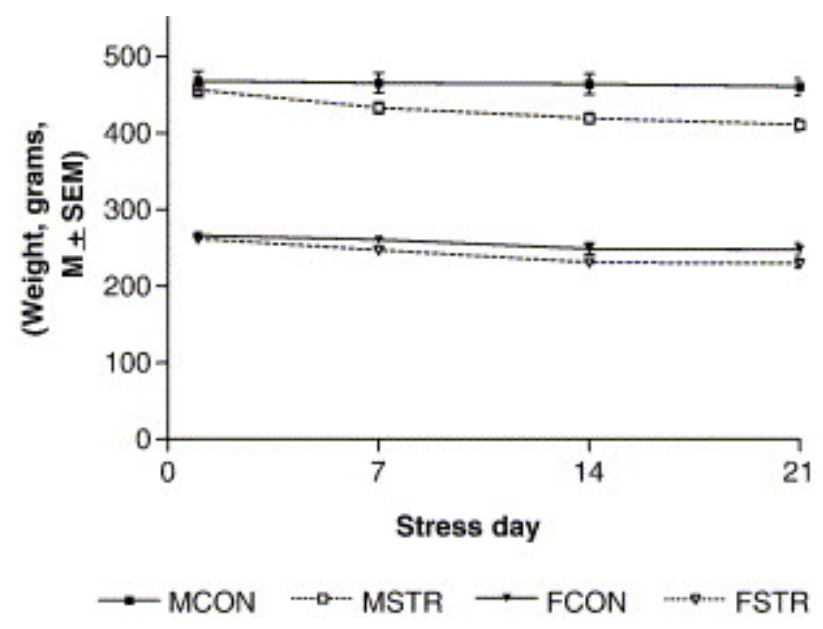

Fig. 1. Body weights for control and stress aged male and females across the 21-day stress period. Body weight during the 21-day stress period was influenced by stress exposure in a sexdependent manner (sex $\times$ treatment $\times$ day $F_{1,161}=13.84, p<0.001$ ). Stress males gained less weight than control males across the 21-day period, but this stress effect across stress duration was less pronounced in stress females compared to controls.

\section{Behavioral measures}

\section{Open field and anxiety-related behavioral indexes}

As shown in Table 1, no significant group differences in overall locomotor activity were found as indicated by either the number of outer sector crossings $(p=0.25)$ or the total number of sector crossings (outer and inner visits combined, $p=0.17$ ). While stressed females were slightly more active (see Table 1), their mean activity scores were not significantly different than the other groups. No group differences were observed with regard to the total number of rears or wall climbs; however, overall, females made significantly more grooms than males (main effect of sex, $\left.F_{1,35}=4.42, p=0.043\right)$. In addition, a significant sex $\times$ treatment interaction $\left(F_{1,35}=4.56\right.$, $p=0.040$ ) was found on the total number of inner sector visits, a measure of anxiety-related behavior. As shown in Table 1, stress increased female inner sector visits (reduced anxiety) but decreased male inner sector visits (increased anxiety). 
Table 1. Summary of open field behavioral measures in control and chronically stressed aged male and female rats

\begin{tabular}{|l|l|l|l|l|l|l|}
\hline Group & Outer visits & Inner visits & Total visits & Rears & Wall climbs & Grooms \\
\hline MCON & $11.8 \pm 1.9$ & $1.90 \pm 0.61^{\times}$ & $13.6 \pm 2.2$ & $1.4 \pm 0.50$ & $7.0 \pm 1.4$ & $5.4 \pm 1.2$ \\
\hline MSTR & $11.5 \pm 1.8$ & $0.60 \pm 0.22^{\times}$ & $12.1 \pm 1.8$ & $1.9 \pm 0.82$ & $6.4 \pm 0.6$ & $6.6 \pm 0.81$ \\
\hline FCON & $12.0 \pm 1.9$ & $0.29 \pm 0.18^{\times}$ & $12.3 \pm 2.0$ & $1.1 \pm 0.83$ & $6.9 \pm 0.88$ & $7.6 \pm 0.95^{\mathrm{a}}$ \\
\hline FSTR & $18.1 \pm 3.6$ & $1.70 \pm 0.89^{\times}$ & $19.8 \pm 4.4$ & $0.55 \pm 0.28$ & $10.5 \pm 1.4$ & $8.0 \pm 0.52^{\mathrm{a}}$ \\
\hline
\end{tabular}

Entries are the mean \pm SEM. A two-factor ANOVA (sex $\times$ stress treatment) with Fischer's LSD post hoc testing was used to test for significant differences among the groups. No significant group differences were observed in overall locomotor activity as measured by either the number of outer sector crossings $(p=0.25$ ) or the total number of sector crossings (outer and inner crossings combined, $p=0.17$ ). No significant effect of sex or stress treatment was observed on rears or wall climbs ( $p \geq 0.05$ ); however, overall, females made more grooms than males (main effect of sex, $\left.F_{1,35}=4.42, p=0.043\right)$. Using inner sector visits as a measure of anxiety, stress decreased female but increased male anxiety (sex $\times$ treatment interaction, $F_{1,35}=4.56$, $p=0.040$ ). Main effect of sex is shown as ${ }^{a}$, and the sex $\times$ treatment interactions is shown as ${ }^{x}$.

In addition to inner sector visits, anxiety-related behavior was measured by the latency to enter the open field and the latency to approach a novel object. While not significant, trends in these anxiety-related behaviors were altered in a sex-specific manner following stress exposure similar to that observed on inner sector visits. Stress increased male latency to enter the field $(\mathrm{MCON}=141.6 \pm 25.1, \mathrm{MSTR}=175.6 \pm 4.4$, data in $\mathrm{s})$ but decreased female latencies $\left(\mathrm{FCON}=158.6 \pm 21.4, \mathrm{FSTR}=123.6 \pm 23.2\right.$, data in $\left.\mathrm{s}, F_{1,35}=2.87, p=0.10\right)$. Group differences in the latency to approach a novel object were present, but these differences did not reach significance $(p=0.13)$. Previous exposure to stress had no effect on females $(F C O N=10.9 \pm 5.2, F S T R=11.0 \pm 2.4$, data in s); however, stress increased male latency to approach the novel object (MSTR $=61.0 \pm 26.0$ ) compared to control males $(\mathrm{MCON}=13.1 \pm 3.3)$ and females, regardless of treatment, $F_{1,35}=2.36, p=0.13$. 


\section{Object recognition}

During the 10 min inter-trial delay, a two-way ANOVA (sex $\times$ treatment) revealed no significant differences between males and females; however, stress effected the amount of time spent exploring both during the sample trial (T1) and during the recognition trial (T2), see Fig. $2 \mathrm{~A}$. During T1, stressed animals spent more time exploring the objects $(5.42 \mathrm{~s})$ than controls (3.45 s), $F_{1,31}=3.94, p<0.05$. Stressed animals continue to explore more during T2, the recognition trial (5.72s), than controls (3.27s), $F_{1,29}=8.13, p<0.008$. However, there were no significant differences among the groups with regard to the percent of time spent with the old versus new object $(p=0.31)$, and all groups had performance at or above chance levels of discrimination during T2, see Fig. 2B.

\section{A. $\quad 10 \mathrm{~min}$ Inter-Trial Delay}

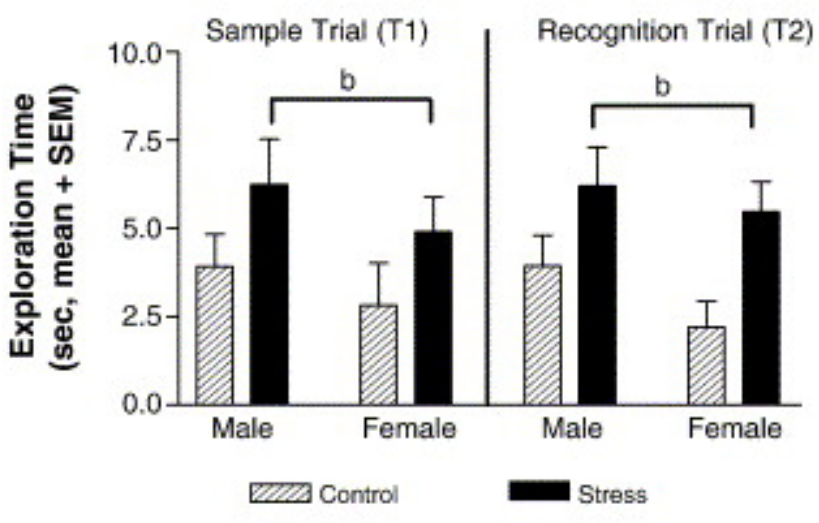

B.

$$
10 \text { min Inter-Trial Delay }
$$

Object Recognition

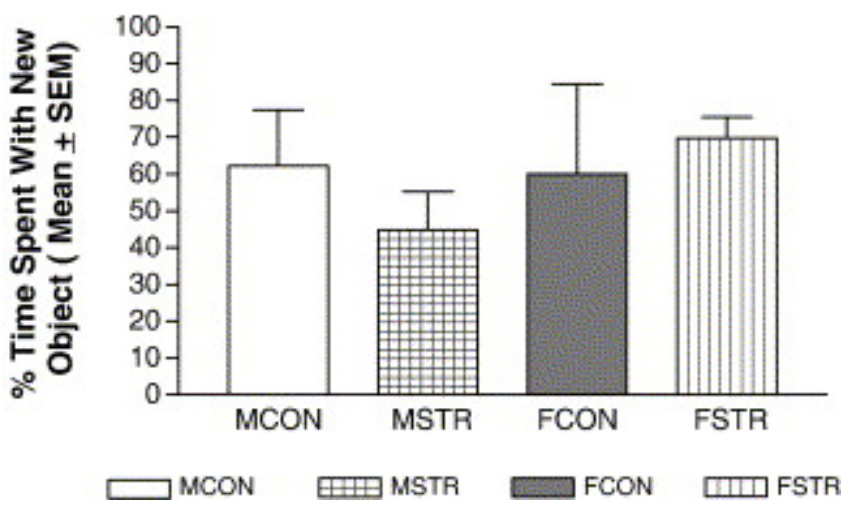

Fig. 2. Stress exposure increases exploration time during a 10 min inter-trial object recognition task. Panel A, data are expressed as seconds (mean \pm SEM) spent exploring both objects for each group during both the sample trial T1 $(\mathrm{MCON}=8, \mathrm{MSTR}=7, \mathrm{FCON}=6, \mathrm{FSTR}=11)$ and the recognition trial T2 $(\mathrm{MCON}=8, \mathrm{MSTR}=6, \mathrm{FCON}=5, \mathrm{FSTR}=11)$. Group differences are identified by the main effect of stress $\left({ }^{b}\right)$. Stress exerted an arousing effect on both males and 
females by increasing the total time spent exploring the objects for stress animals during both T1 $(p=0.05)$ and T2 $(p=0.01)$. In panel B, data are expressed as the percent of total T2 time spent with the new object (mean $\pm \mathrm{SEM}$ ) for each group $(\mathrm{MCON}=8, \mathrm{MSTR}=6, \mathrm{FCON}=5$, FSTR = 11). While there were no significant group differences, all animals performed at or above chance levels indicating an ability to discriminate between the old and new objects.

Stress subjects continued to spend more time exploring the objects (stress $=6.08 \mathrm{~s}$ ) during the sample trial on the $1 \mathrm{~h}$ inter-trial delay trial as compared to controls $(2.74 \mathrm{~s}), F_{1,31}=7.25$, $p=0.011$, see Fig. 3A. No significant effects of either sex $(p=0.14)$ or stress $(p=0.13)$ on total exploration following the $1 \mathrm{~h}$ inter-trial delay (T2) were found, Fig. 3A. While none of the groups successfully discriminated between the old and new objects during $\mathrm{T} 2$, there was a main effect of stress on the discrimination index, $F_{1,30}=4.35, p=0.046$, and stressed animals spent more time with the new object (47\%) than controls (25\%), see Fig. 3B. With a longer inter-trial delay $(2 \mathrm{~h})$, no effects of sex, stress or interactions on performance were found in T1 or T2 (data not shown). 


\section{A. $\quad 1 \mathrm{hr}$ Inter-Trial Delay \\ Object Recognition}
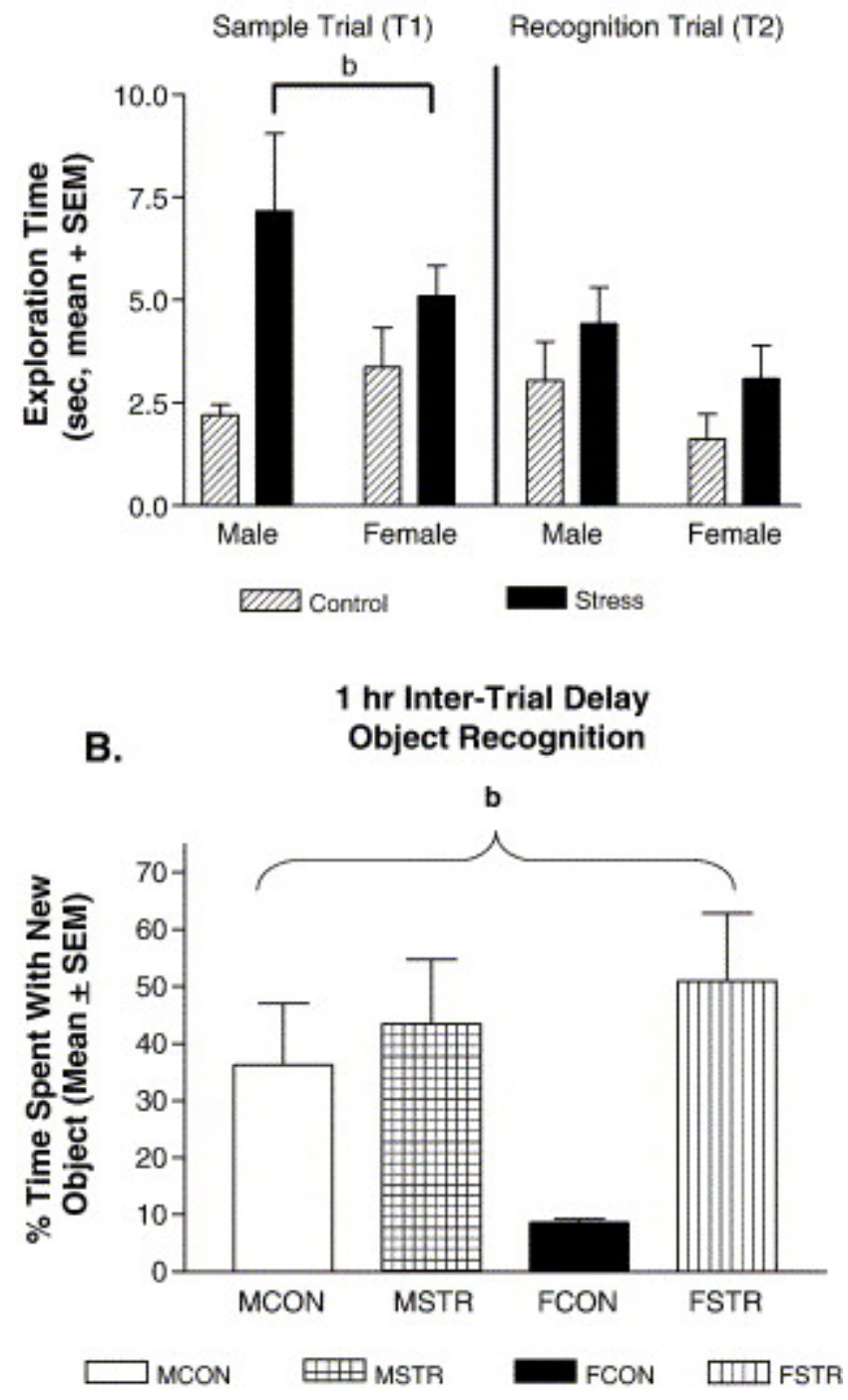

Fig. 3. Stress, but not sex, alters performance on a $1 \mathrm{~h}$ inter-trial object recognition task. In panel $A$, data are expressed as seconds (mean \pm SEM) spent exploring for each group during both the sample trial T1 $(\mathrm{MCON}=7, \mathrm{MSTR}=9, \mathrm{FCON}=6, \mathrm{FSTR}=10)$ and the recognition trial T2 $($ MCON = 7, MSTR = 9, FCON = 5, FSTR = 10). Group differences are identified by the main effect of stress treatment (b). Stress increased exploration during T1 ( $p=0.012$, but not during T2 $(p=0.13)$. In panel $B$, data are expressed as the percent of total T2 time spent with the new object (mean \pm SEM) for each group $(M C O N=7, M S T R=9, F C O N=5, F S T R=10)$. None of the groups spent significantly more than $50 \%$ of their time with the novel object, and this is considered to be below chance levels of discrimination. Nonetheless, there was a main effect of stress (b) and stress animals spent more time with the new object following a $1 \mathrm{~h}$ inter-trial delay than the control animals $(p=0.046)$. 


\section{Object placement}

For the $1 \mathrm{~h}$ inter-trial delay object placement task, exploration times during the sample trial T1 were longer in females $(n=17, \sim 13 \mathrm{~s})$ as compared to males $(n=18, \sim 7 \mathrm{~s})$, main effect of $\operatorname{sex}\left(F_{1,34}=10.5, p=0.002\right)$. Initial exploration time (T1) was not influenced by stress exposure $(p=0.59)$ or the sex by treatment interaction $(p=0.57)$. Exploration time following the $1 \mathrm{~h}$ intertrial delay T2 was not influenced by sex $(p=0.88)$, stress $(p=0.28)$ or the interaction $(p=0.35)$. As measured by the discrimination ratio (Fig. 4), none of the groups was able to successfully discriminate between the old and new locations. In fact, all groups spent less than $50 \%$ of their time with the new location: MCON $=45 \%(n=8), \operatorname{MSTR}=33 \%(n=10)$, FCON $=45 \%(n=7)$, $\operatorname{FSTR}=29 \%(n=11)$.

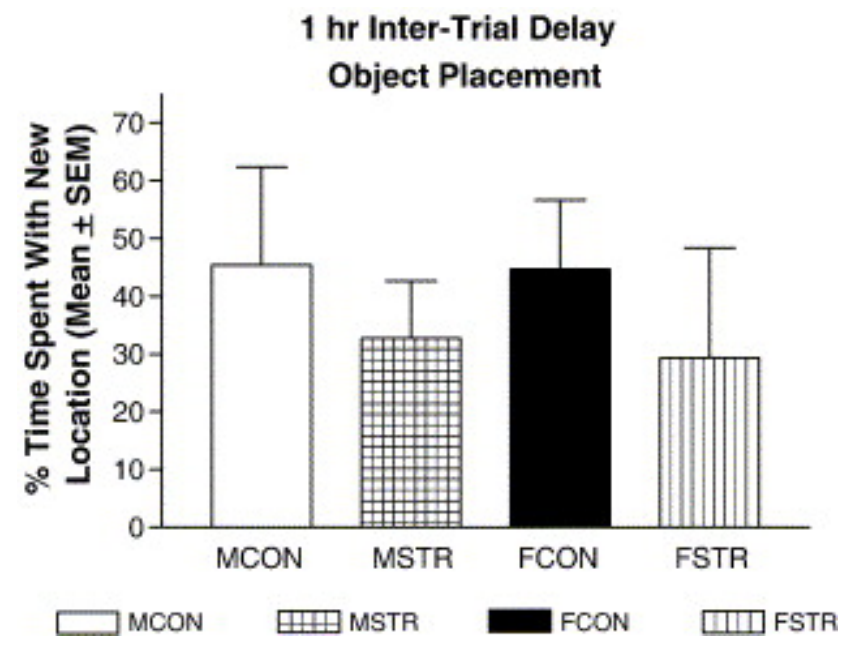

Fig. 4. Aged male and female rats have impaired spatial memory performance on the object placement task, and there is no sex difference. Data are expressed as the percent of total T2 time spent with the object in the new location (mean \pm SEM) for each group (MCON = 8, MSTR $=10$, FCON $=7$, FSTR $=11$ ). None of the groups spent significantly more than $50 \%$ of their time with the object in the new location, and this is considered to be below chance levels of discrimination.

\section{Hormone measurements}

Serum levels of CORT, estradiol, and T were obtained at sacrifice, 10 days after the last restraint session. Serum levels of CORT are shown in Fig. 5A. CORT levels were significantly higher in females than males, main effect of $\operatorname{sex}\left(F_{1,31}=4.7, p<0.038\right)$, but no difference in control and stress subjects. Serum levels of estradiol $\left(E_{2}\right)$ in the aged males and females are shown in Fig. 5B. A significant effect of sex on $E_{2}$ was observed $\left(F_{1,31}=9.3, p<0.005\right)$, and aged females, regardless of treatment, had lower levels of $E_{2}$ than the males. Chronic stress had no sustained effect on $E_{2}$ levels in either males or females. Serum levels of $T$ in the aged males and females are shown in Fig. 5C. As expected, levels of $\mathrm{T}$ were substantially higher in 
males than in females (main effect of sex, $F_{1,31}=31.48, p<0.001$ ). No significant enduring effects of chronic stress $(p>0.05)$ on T levels were found in either males or females.
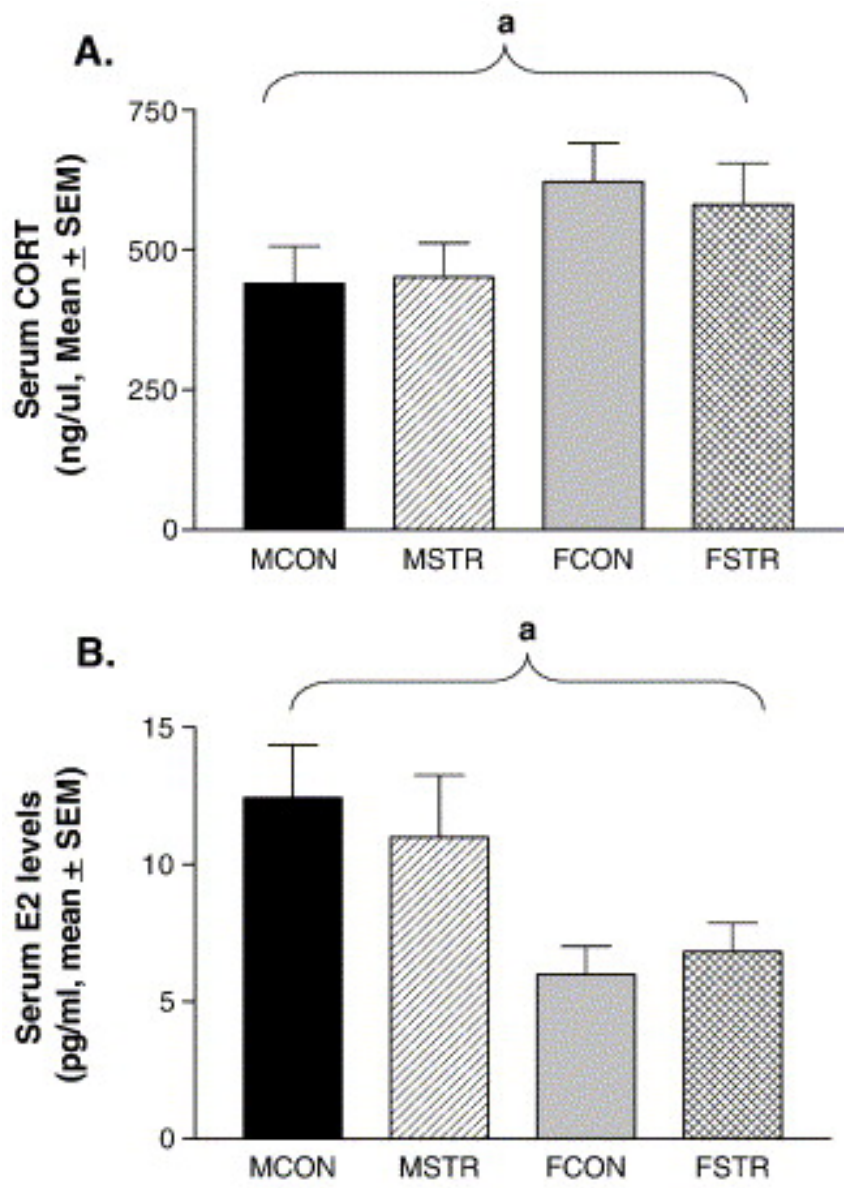

C.

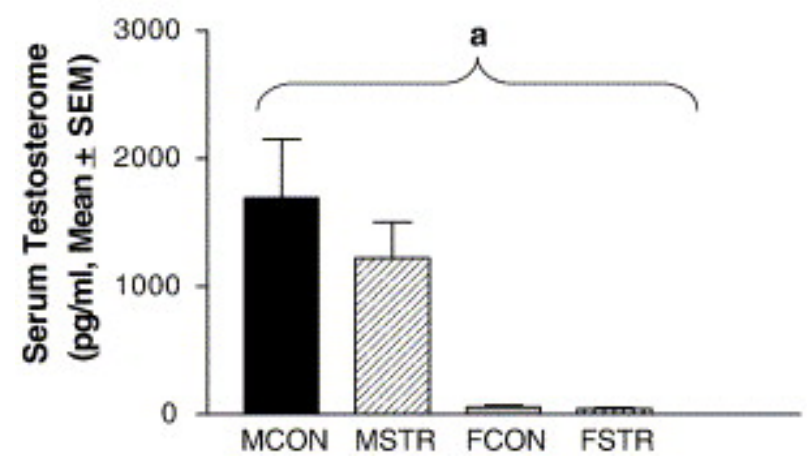

Fig. 5. Serum levels of CORT, estradiol and testosterone are influenced by sex, but not stress. Entries are the mean \pm SEM for each group for CORT (A), estradiol (B) and testosterone (C). Group differences are identified by the main effect of sex (a). Aged females had higher levels of serum CORT than males $(p<0.038)$. Aged males had higher levels of both serum estradiol $(p<0.005)$ and testosterone $(p<0.001)$ than females. 


\section{DISCUSSION}

\section{Physiological measurements}

\section{Weight and hormone levels}

Aged animals did not gain weight during the course of the study, and this result is consistent with previous reports (Black et al., 2003), but different from results in young rats (e.g., Bowman et al., 2001, 2002, 2004). Importantly, the current data provide novel information regarding body weights across time in aged females, an area that has received little attention in the past. Aged animals lost weight in response to stress in a similar fashion to that previously observed for young animals (Bowman et al., 2001, Gregus et al., 2005 and Magarinos and McEwen, 1995), that is weight loss is more pronounced in stress males than stress females. While the endocrine underpinnings of this sex difference in weight alterations in response to stress remain to be determined, possible mechanisms include higher levels of testosterone in males (Gabriel et al., 1992) than females, an observation which persists even in aged rats.

The aged rats appeared to be relatively resistant to chronic stress in terms of enduring effects on both CORT and gonadal steroid levels. As expected, chronic stress was not associated with a significant increase in serum CORT levels 10 days after cessation of stress. These findings are similar to past research with young animals which have demonstrated that stress-induced CORT levels decline during the course of chronic restraint stress (Bowman et al., 2001 and Galea et al., 1997) and are no longer present following behavioral testing (Bowman et al., 2001). Thus, the delayed termination of stress response in aged animals that has previously been reported (e.g., Bodnoff et al., 1995) appears to be temporally constrained and does not extend to durations of 10 days post-stress. While sex differences in HPA activity as measured by serum CORT levels have been widely examined in young adults, there are less data concerning possible sex differences in aged animals and here we show that that aged females continue to have elevated levels of CORT in comparison to aged males. It appears that sex differences in HPA axis activity that are established following gonadal steroid exposure during the organizational period (e.g., Patchev et al., 1995) are maintained in adulthood when the activational influences of hormones on HPA activity have decreased.

Although previous studies in young animals have demonstrated a significant inhibitory effect of chronic stress on circulating testosterone concentrations (Gao et al., 2002, Monder et al., 1994 and Orr and Mann, 1992) as long as 14 days following stress exposure (Hardy et al., 2002), no significant effect of stress on gonadal steroid levels was observed. As expected, the levels of estradiol in old females were relatively low, approximating the levels observed at the nadir of the normal estrous cycle (Scharfman et al., 2003). Previous work has shown that, as female rats age, they gradually lose the capacity to support estrous cycles and begin, at around 18 months to 2 years of age, to exhibit persistent diestrus (for a review, see Hung et al., 2003). The estradiol levels measured in the present group of aged females are consistent with these previous findings. In old males, however, we observed surprisingly high serum estradiol concentrations, approximately $12 \mathrm{pg} / \mathrm{ml}$, twice those observed in females at the same age and more than 4 times higher than the assay detection limit and close to the levels observed normally in females during the early follicular phase of the estrous cycle (Edwards et al., 1999). 
This results contrasts with gonadal hormone levels in young males, in which estradiol levels are typically similar to those observed in anestrous females and close to the limit of sensitivity of the assay used in this study (Edwards et al., 1999). The origin of the circulating estradiol detected in old males remains to be established. Circumstantial evidence points to the possibility of peripheral conversion of circulating testosterone. Thus, while stress did not significantly affect levels of either testosterone or estradiol individually, the levels of both hormones tended to decrease as might be expected if the estrogen was derived from peripheral metabolism of androgen. In primates, fat represents a significant source of peripheral aromatase activity. As rats age, they accumulate increasing amounts of body fat (Thomas et al., 2002). A reasonable hypothesis, therefore, is that increased levels of circulating estradiol in aging male rats might reflect rising levels of extragonadal aromatase activity.

\section{Behavioral measurements}

\section{Anxiety-related behaviors and locomotion}

Male and female anxiety-related behavioral levels and the effects of stress exposure on these measurements in aged male and female rats presented in the current study are different from those previously observed in young rats. Past research has demonstrated that young control female rats are generally less anxious than young control males (e.g., latency to enter open field, Beck and Luine, 2002; Bowman et al., 2004), however, exposure to chronic restraint stress exerts anxiogenic effects on young females (increases entry latency), but anxiolytic effects on young males (decreases entry latency) (Beck and Luine, 2002, Bowman et al., 2004 and Brown and Grunberg, 1995). Thus, sexually differentiated chronic stress effects on anxiety-related behavioral levels appear reversed during aging.

One possibility for the difference in stress effects for young vs. aged rats on anxiety-related behavioral levels could be due to estrogen levels declining in females and increasing in males with aging. While results have been mixed, estrogen has been shown to decrease anxietyrelated behavioral measures using the open field and the elevated plus maze (Bowman et al., 2002, Mora et al., 1996 and Nomikos and Spyraki, 1988), and it is possible that as female estrogen levels decline with aging there is an increase in overall anxiety-related behavioral levels. Other mechanisms for sex-specific changes in anxiety-related behavioral responses to stress in aged rats (e.g., CRH; Magiakou et al., 1997 and Steckler and Holsboer, 1999) may be pertinent and require further investigation.

There were no group differences in overall activity, as measured by locomotor activity on the open field. This result is similar to other reports that have not found changes in open field activity levels following either repeated CORT injections or restraint stress (Bowman et al., 2001; Brotto et al., 2001; Gregus et al., 2005). Thus, it is unlikely that any subsequent sex or stress group differences on either the object recognition or object placement task are due to differences in activity levels. 


\section{Object recognition}

Young rats can successfully discriminate between the old and new objects with an object recognition inter-trial delay of $4 \mathrm{~h}$, and there is no sex difference in object recognition performance (Beck and Luine, 2002 and Ennaceur and Agleton, 1997). Here, there was also no observed sex difference in performance in 21.5-month-old aged rats. All groups appeared able to discriminate between the old and new objects at the 10 min interval, and this result is similar to previous reported results of intact novel object recognition in male and female aged mice (Benice et al., 2006). However, performance dropped to below chance levels of discrimination following the $1 \mathrm{~h}$ inter-trial delay. The longer inter-trial delay imposes greater cognitive demand on the animal and, thus, while aged animals can generally discriminate following the short delay, they cannot perform as well as young animals (who can discriminate at the $4 \mathrm{~h}$ inter-trial delay). Notable also is the observation that, while object discrimination ability was impaired for all groups following the $1 \mathrm{~h}$ inter-trial delay, there was a significant effect of stress exposure on the discrimination index. Specifically, stress increased the percentage of time spent with the new object (47\%) almost double the percentage of time spent by controls (25\%). Because aged rats are known to have an increase in perseverative errors (Colombo and Gallagher, 1998), it appears possible that the low index ratio of percent time spent with new object in controls (25\%) is indicative of perseveration for the familiar object. However, stress exposure appeared to attenuate this perseveration effect, and similar results have been observed in young male and female rats that spent less time with a familiar stimulus following acute stress (Shors and Wood, 1995).

Stress did not affect male performance on the object recognition task, and these results are different from young males who have impaired performance following chronic stress (Beck and Luine, 2002), see Fig. 3. Estrogens are known to influence female resistance to deleterious stress effects on cognitive performance (Bowman et al., 2002) and, thus, a reasonable hypothesis is that the elevated levels of E2 observed in the aged males are conferring stress resistance on performance (for a review, see Bowman, 2005). It appears that aged male rats are responding to restraint stress exposure in a similar fashion to young females who are thought to be protected from the deleterious effects of stress by their ovarian hormones. Additionally, recent studies have shown that testosterone improves working memory in aged rats (Bimonte-Nelson et al., 2003). In the current study, exposure to chronic stress did not reduce $T$ which may have contributed to ability of both aged control and aged stress males to perform the object recognition task. Aged males have both testosterone and elevated estradiol and, thus, these gonadal hormones may be providing a buffering effect of the deleterious effects of stress in object recognition performance.

Aged control females cannot perform the object recognition task (i.e., discrimination ratios are chance, 50\%), and this observation is different from young females who are able to successfully discriminate between old and new objects (Beck and Luine, 2002); however, aged female impairments are mitigated by exposure to stress, that is, stressed aged females can discriminate between objects. In young females, stress does not affect OR performance (Beck and Luine, 2002). Thus, it appears that in old, but not young, female rats chronic stress enhances object recognition performance. The underlying mechanisms for chronic stress effects 
on cognitive function in young and old females are unclear but may include changed sensitivities of the HPA axis and changes in gonadal hormone levels in the aged females (for comments on the influence of age on sex-dependent changes following stress exposure, see Bowman, 2005).

\section{Object placement}

For the object placement task, a clear sex difference in the performance of young animals has previously been observed with males typically discriminating between the old and new location following object placement tests with inter-trial delays up to $4 \mathrm{~h}$, while females can only discriminate following inter-trial delays of 1-2 h (Beck and Luine, 2002; for a review, see Bowman et al., 2003). This sex difference is present in other spatial tasks such as the radial arm maze and Morris water maze (Roof, 1993 and Kitraki et al., 2004). In the current study, all aged subjects were impaired, regardless of sex or treatment, following an inter-trial delay of $1 \mathrm{~h}$ (Fig. 4). Groups spent less than $50 \%$ of their time with the object in the new location, below chance levels of discrimination. Impaired spatial memory performance in our aged subjects is consistent with numerous other reports that have shown declines in spatial learning and memory in aged animals [for a review, see Barnes, 1988 and Gallagher and Pelleymounter, 1988].

Perhaps most importantly, the current results show that the normal male advantage seen on tasks of spatial learning and memory dissipates with age (that is, there was no performance difference in the control males and females). These results are similar to other findings that show that aging impairs spatial learning on the Morris water maze and mitigates the standard sex difference in favor of males on spatial tasks (Veng et al., 2003). The amelioration of the typical male spatial advantage appears to be due to a more pronounced effect of aging on male, than female, performance in both rats (Veng et al., 2003) and rhesus monkeys (Lacreuse et al., 2005). It has recently been shown that dendritic morphology in the CA1 region of the hippocampus is sexually dimorphic during aging, with male atrophy more severe than female changes (Markham et al., 2005), and this could account for the similar performance of males and females in the current study.

\section{Conclusions}

Taken together, the current results offer additional support to the notion that sex differences in spatial memory are less pronounced in aged animals and provide novel data that aged rats are less sensitive/responsive to chronic restraint stress effects on both spatial and non-spatial learning and memory performance than what has previously been reported for young rats (for a review, see Bowman, 2005). Thus, aging is associated with alterations in cognitive responses to stress that are different from those observed in young animals (that is, females are no longer enhanced and males are unaffected by stress) and that the standard sex differences in spatial memory are no longer present in aged animals. However, it should be noted that this nonresponsivity may be due to low levels of performance in the non-stressed, aged animals. It 
remains to be determined if the current results hold true for other spatial and non-spatial memory tasks, and future studies are necessary to elucidate whether the observed behavioral changes are due to changes in memory per se or rather impairments in learning. Additionally, there is a clear need for future studies to conduct a direct comparison between young and various aged animals to determine when cognitive functioning (under both non-stressed and stressed conditions) is altered and to examine possible underlying mechanisms. In young adult rats, changes in anxiety-related behaviors and cognitive function (both spatial and non-spatial memory) are accompanied by sex-dependent alterations in central monoamine and metabolites levels in brain areas related to these functions (e.g., stress increases frontal cortex dopamine levels in females compared to males, for a review, see Bowman et al., 2003). Because limited information is available concerning stress and/or aging effects in females, it remains to be determined if changes in monoamine levels during aging are influencing the cognitive changes observed here in the aging stress/non-stress animals.

The chronic restraint stress model in rats is useful because the observed effects may mimic the abnormalities observed in humans exposed to periods of stress (Wolf, 2003). In addition, increased CORT secretion is associated with a variety of age-related disorders such as Alzheimer's disease, depression, diabetes, and hypertension. Because the U.S. population continues to age dramatically, with females outliving males, there is a clear need to determine the influence of sex on stress, aging, and cognitive function and possible underling mechanisms for these complex interactions. Thus, sex/gender must be given greater consideration in these issues.

\section{EXPERIMENTAL PROCEDURE}

\section{Subjects}

Aged, 20-month-old male $(n=20)$ and female $(n=20)$ Fischer 344 rats were obtained from the National Aging Institute aged rat colony (maintained by Harlan Sprague-Dawley, Inc). All animals were maintained on a 14/10-h light/dark cycle (lights on 5:00 am). All experimental procedures were approved by the Hunter College Institutional Animal Care and Use Committee and in accordance with the NIH Guide for the Care and Use of Animals. All animals were double housed in same sex pairs and had free access to rat chow and water.

Following a two-week acclimation period during which animals were allowed adjust to the new housing conditions, subjects were randomly assigned to either a control $(n=8$ males [MCON] and $n=8$ females [FCON]) or stressed ( $n=12$ males [MSTR] and $n=12$ females [FSTR]) condition. Experimental subjects were exposed to chronic restraint stress for $6 \mathrm{~h} /$ day for 21 consecutive days as previously applied in this (Beck and Luine, 2002, Bowman et al., 2001, Bowman et al., 2002 and Luine et al., 1996) and other laboratories (Conrad et al., 1996, Conrad et al., 2004, Kitraki et al., 2004, McLaughlin et al., 2005 and Nacher et al., 2004). The restraint stress was applied by placing each rat in a Plexiglas tube (Harvard Apparatus ${ }^{\mathrm{TM}}$ ). The Plexiglas 
restraint tubes are equipped with air holes and an adjustable endplate, which helps account for differences in body weight and length and secures the rat within the tube. Once the rats were secured in their respective Plexiglas tube, the cohort was placed in a ventilated isolation chamber located in a separate room from the colony. Control animals received equal amounts of daily handling, approximately 1-2 min in the morning and afternoon, but remained undisturbed in their home cages during the stress duration. All animals were weighed weekly. Four animals (FCON $=1, F S T R=1, M S T R=2)$ died during the course of the study, and their data were not included in the analyses.

\section{Behavioral measures}

On the day following the stress period, all subjects (now 21.5 months old) began behavioral testing procedures. Open field was conducted first followed by object recognition testing and then object placement testing. All behavior was identically run for two cohorts of animals, and all behavioral testing occurred between 9:00 am and 2:00 pm.

\section{Open field and anxiety-related behavioral indexes}

\section{Open field}

On post-stress day 1 , overall locomotor activity was assessed using the open field paradigm. Rats were placed in a $15 \times 15 \mathrm{~cm}$ small box with a sliding door opening to the corner of the open field; the open field was $117 \times 70 \times 45 \mathrm{~cm}$ black, Plexiglas, open top box, with the floor divided into 9 inch square grids $(5 \times 3)$. The sliding door was opened and latency to enter the field scored (an anxiety-related behavior). Each animal was allowed $3 \mathrm{~min}$ to enter the field voluntarily. If the animal failed to enter the field, they were hand-placed inside the field and a latency time of $180 \mathrm{~s}$ was recorded. Number of outer sector visits (movements across squares and a measure of general locomotor activity), inner sector visits (an anxiety-related behavior), total activity (combined outer and inner crossings), wall climbs, rears and grooms were scored for 6 min. After each animal, the open field was wiped down with disinfectant spray to minimize olfactory cues.

\section{Novel object exposure}

After all animals had completed the open field test, animals individually were tested on latency to approach a novel object (post-stress day 1). Animals were placed in the center of the open field, and the time to approach and explore a novel object located at the end of the field was recorded. Exploration of the novel object (Johnson's Baby Oil or Poland Spring Water Bottle, both $8 \mathrm{oz}$. randomized across subjects) was defined as the subject sniffing at, whisking at or looking at the object from no more than $2 \mathrm{~cm}$ away. Each animal was allowed $3 \mathrm{~min}$ to approach 
and explore the novel object. If the animal failed to approach and explore the novel object, an exploration latency time of $180 \mathrm{~s}$ was recorded.

\section{Object recognition}

Recognition memory was assessed using the object recognition (OR) task as previously described in young rats (Beck and Luine, 2002 and Bowman et al., 2004). Trials consisted of a sample trial (T1) and a recognition trial (T2). The two trials were separated by an inter-trial interval of varying lengths. In T1, two identical objects were placed at one end of the open field and amount of time spent exploring the two objects was recorded for 3 min. For T2, or the recognition trial, a new distinct object replaced one of the previous objects. In T2, the time spent exploring the old (familiar object) and the new (novel) object was recorded for 3 min. Thus, the percentage of time spent with the novel object during the total exploration time during T2 is used as an index of object recognition. Exploration was defined as when the subject sniffed at, whisked at or looked at the object from no more than $2 \mathrm{~cm}$ away. The objects used for trials were various bottles, cans and containers, and the novel object was counterbalanced across both sex and treatment. All animals received pre-testing acclimation session with an inter-trial delay of $1 \mathrm{~min}$ (post-stress day 2) and were then tested for object recognition with a 10 min (post-stress day 3), $1 \mathrm{~h}$ (post-stress day 4 ) and $2 \mathrm{~h}$ inter-trial delay (post-stress day 5). Animals that did not explore the objects during either T1 or T2 were not included in the data analysis (group sample sizes are referred to in corresponding figure legend).

\section{Object placement}

Spatial memory was assessed using the object placement (OP) task, a spatial memory task known to be hippocampal-dependent and that has been used previously with young rats (Beck and Luine, 2002 and Li et al., 2004). This task is identical to the OR procedure, except that during T2, the recognition trial, one object is moved to a novel location within the field (instead of replacing one object with a new object). Time spent by each subject exploring objects at the old versus new location was recorded during T2. All animals received a $10 \mathrm{~min}$ acclimation trial (post-stress day 8) and were then tested on a $1 \mathrm{~h}$ inter-trial delay (post-stress day 9). The new location was counterbalanced across sexes and treatments. Animals that did not explore the objects during either T1 or T2 were not included in the data analysis (group sample sizes are referred to in corresponding figure legends).

\section{Hormone assays}

Immediately following behavioral testing, 10 days post-stress, all subjects were sacrificed via rapid decapitation and trunk blood was collected for subsequent radioimmunoassay. Blood was allowed to separate at room temperature; serum was collected after centrifugation in a clinical centrifuge and frozen in sealed tubes at $-80{ }^{\circ} \mathrm{C}$ until assay. 
Total serum CORT levels were measured by radioimmunoassay using the Coat-A-Count ${ }^{\circledR}$ assay kit, according to kit protocol, available from Diagnostic Products Corporation, Los Angeles, CA (catalog number TKRC1). Samples were analyzed in duplicates. All samples were analyzed simultaneously, and the detection limit was $5.7 \mathrm{ng} / \mathrm{ml}$, as defined by the $95 \%$ confidence limits of the zero standard. CORT levels are expressed as $\mathrm{ng} / \mathrm{ml}$.

Serum testosterone ( $T$ ) levels were measured by RIA using commercial kits (Coat-A-Count kits Cat \#TKTT1, Diagnostic Product Corporation, Los Angeles, CA) according to the kit instructions. Serum sample volumes were $50 \mu \mathrm{l}$. To correct for any potential blank effect due to components of rat serum, the standards for the assay were prepared in rat serum from a pool obtained from ovariectomized animals. The minimum detectable concentration of hormone was $0.15 \mathrm{ng} / \mathrm{ml}$.

Serum $E_{2}$ levels were also measured using commercial RIA kits (Coat-A-Count RIA kit KE2D1; Diagnostic Products Corp, Los Angeles, CA). Using this kit, which is designed for use with human samples, according to the manufacturer's instructions, estradiol levels are overestimated as a result of interference from rat serum binding proteins (MacLusky, unpublished observations). Correction for this interference cannot entirely be achieved by adding an equivalent volume of serum from ovariectomized rats to the assay standards. However, the interference can be minimized by organic solvent extraction to partially purify the estradiol prior to assay. The assay standards and rat serum samples (100 $\mu \mathrm{l} / \mathrm{sample})$ were therefore extracted prior to assay with $2 \mathrm{ml}$ anhydrous ethyl ether. The ether extracts were rapidly dried under a stream of air, reconstituted into $100 \mu \mathrm{l}$ of the human serum zero calibration standard from the RIA kit and assayed according to kit instructions. The minimum detectable concentration of estradiol was $3 \mathrm{pg} / \mathrm{ml}$. All samples for both testosterone and estradiol measurement were run on the same day.

\section{Data analysis}

Animal body weights were analyzed using a two-way ANOVA, sex (male, female) $\times$ treatment (control, stress) with stress day as a repeated measure. Two-way ANOVAs, sex (male, female) $\times$ treatment (control, stress), were used to test for statistical group differences on the open field and anxiety-related behavioral measures. Two-way ANOVAs (sex $\times$ stress treatment) were used to test for group differences on the time spent exploring objects during the sample trial (T1), the time spent exploring the objects during the recognition trial (T2) and in the discrimination between old and new objects (for non-spatial memory) or locations (for spatial memory) during T2 (ratio) for all object recognition and object placement trials. Additional twoway ANOVAs, sex (male, female) $\times$ treatment (control, stress), were used to test for statistical group differences in corticosterone, testosterone and estrogen levels at sacrifice. Type I error rate was set at 0.05 and significant ANOVA results were followed by Fisher's LSD post hoc tests. 


\section{ACKNOWLEDGMENTS}

Funding support was provided by SHU URI grant to SED and REB and NIH grants GM60654, DA12136 and GI2RR3037 to VNL. We thank Govini Mohan and Tomoko Inagaki for their assistance with behavioral data collection.

\section{REFERENCES}

Adams et al., 2001 M.M. Adams, R.A. Shah, W.G.M. Janssen and J.H. Morrison, Different modes of hippocampal plasticity in response to estrogen in young and aged female rats, Proc. Natl. Acad. Sci. 98 (2001) (14), pp. 8071-8076.

Adams et al., 2002 M.M. Adams, S.E. Fink, R.A. Shah, W.G.M. Janssen, S. Hayashi, T.A. Milner, B.S. McEwen and J.H. Morrison, Estrogen and aging affect the subcellular distribution of estrogen receptor- $\alpha$ in the hippocampus of female rats, J. Neurosci. 22 (2002) (9), pp. 36083614.

Astur et al., 2004 R.S. Astur, J. Tropp, S. Sava, R.T. Constable and E.J. Markus, Sex differences and correlations in a virtual Morris water task, a virtual radial arm maze, and mental rotation, Behav. Brain Res. 151 (2004), pp. 103-115.

Barnes, 1988 C.A. Barnes, Aging and the physiology of spatial memory, Neurobiol. Aging 9 (1988), pp. 563-568.

Beck and Luine, 2002 K.D. Beck and V.N. Luine, Sex differences in behavioral and neurochemical profiles after chronic stress: role of housing conditions, Physiol. Behav. 75 (2002), pp. 661-673.

Benice et al., 2006 T.S. Benice, A. Rizk, S. Kohama, T. Pfankuch and J. Raber, Sex differences in age-related cognitive decline in C57BL/6 J mice associated with increased brain microtubuleassociated protein 2 and synaptophysin immunoreactivity, Neuroscience 137 (2006) (2), pp. 413-423.

Bimonte-Nelson et al., 2003 H.A. Bimonte-Nelson, R.S. Singleton, M.E. Nelson, C.B. Eckman, J. Barber, T.Y. Scott and A.E. Granholm, Testosterone, but not nonaromatizable dihydrotestosterone, improves working memory and alters nerve growth factor levels in aged male rats, Exp. Neurol. 181 (2003), pp. 301-312.

Black et al., 2003 B.J. Black Jr., C.A. McMahan, E.J. Masoro, Y. Ikeno and M.S. Katz, Senescent terminal weight loss in the male F344 rat, Am. J. Physiol.: Regul., Integr. Comp. Physiol. 284 (2003), pp. 336-342.

Bodnoff et al., 1995 S.R. Bodnoff, A.G. Humphreys, J.C. Lehman, D.M. Diamond, G.M. Rose and M.J. Meaney, Enduring effects of chronic corticosterone treatment on spatial learning, synaptic plasticity, and hippocampal neuropathology in young and mid-aged rats, J. Neurosci. 15 (1995), pp. 61-69. 
Bowman, 2005 R.E. Bowman, Stress-induced changes in spatial memory are sexually differentiated and vary across the lifespan, J. Neuroendocrinol. 17 (2005) (8), pp. 526-535.

Bowman et al., 2001 R.E. Bowman, M.C. Zrull and V.N. Luine, Chronic restraint stress enhances radial arm maze performance in female rats, Brain Res. 904 (2001), pp. 279-289.

Bowman et al., 2002 R.E. Bowman, D. Ferguson and V.N. Luine, Effects of chronic restraint stress and estradiol on open field activity, spatial memory, and monoaminergic neurotransmitters in ovariectomized rats, Neuroscience 113 (2002), pp. 401-410.

Bowman et al., 2003 R.E. Bowman, K.D. Beck and V.N. Luine, Chronic stress effects on memory: sex differences in performance and monoaminergic activity, Horm. Behav. 43 (2003), pp. 48-59.

Bowman et al., 2004 R.E. Bowman, N.J. MacLusky, Y. Sarmiento, M. Frankfurt, M. Gordon and V.N. Luine, Sexually dimorphic effects of prenatal stress on cognition, hormonal responses and central neurotransmitters, Endocrinology 145 (2004), pp. 3778-3787.

Brotto et al., 2001 L.A. Brotto, B.B. Gorzalka and A.M. Barr, Paradoxical effects of chronic corticosterone on forced swim behaviours in aged male and female rats, Eur J. Pharmacol 424 (2001), pp. 203-209.

Brown and Grunberg, 1995 K.J. Brown and N.E. Grunberg, Effects of housing on male and female rats: crowding stresses males but calms females, Physiol. Behav. 58 (1995), pp. 10851089.

Bucci et al., 1995 D.J. Bucci, A.A. Chiba and M. Gallagher, Spatial learning in male and female Long-Evans rats, Behav. Neurosci. 109 (1995), pp. 180-183.

Chen et al., 1994 H. Chen, M.P. Hardy, I. Huhtaniemi and B.R. Zirkin, Age-related decreased Leydig cell testosterone production in the Brown Norway rat, J. Androl. 15 (1994), pp. 551-557.

Colombo and Gallagher, 1998 P.J. Colombo and M. Gallagher, Individual differences in spatial memory and striatal ChAT activity among young and aged rats, Neurobiol. Learn. Mem. 70 (1998) (3), pp. 314-327.

Conrad et al., 1996 C.D. Conrad, L.A. Galea, Y. Kuroda and B.S. McEwen, Chronic stress impairs rat spatial memory on the $Y$ maze, and this effect is blocked by tianeptine pretreatment, Behav. Neurosci. 110 (1996), pp. 1321-1334.

Conrad et al., 2003 C.D. Conrad, K.A. Grote, R.J. Hobbs and A. Ferayorni, Sex differences in spatial and non-spatial Y-maze performance after chronic stress, Neurobiol. Learn. Mem. 79 (2003), pp. 32-40.

Conrad et al., 2004 C.D. Conrad, J.L. Jackson and L.S. Wise, Chronic stress enhances ibotenic acid-induced damage selectively within the hippocampal CA3 region of male, but not female rats, Neuroscience 125 (2004), pp. 759-767. 
Driscoll et al., 2005 I. Driscoll, D.A. Hamilton, R.A. Yeo, W.M. Brooks and R.J. Sutherland, Virtual navigation in humans: the impact of age, sex, and hormones on place learning, Horm. Behav. 47 (2005), pp. 326-335.

Edwards et al., 1999 H.E. Edwards, W.M. Burnham and N.J. MacLusky, Partial and generalized seizures affect reproductive physiology differentially in the male rat, Epilepsia 40 (1999), pp. 1490-1498.

Ennaceur and Agleton, 1997 A. Ennaceur and J.P. Agleton, The effects of neurotoxic lesions of the perirhinal cortex combined to fornix transaction on object recognition memory in the rat, Behav. Brain Res. 88 (1997), pp. 181-193.

Gabriel et al., 1992 S.M. Gabriel, J.R. Roncancio and N.S. Ruiz, Growth hormone pulsatility and the endocrine milieu during sexual maturation in male and female rats, Neuroendocrinology 56 (1992), pp. 619-625.

Galea et al., 1997 L.A. Galea, B.S. McEwen, P. Tanapat, T. Deak, R.L. Spencer and F.S. Dhabhar, Sex differences in dendritic atrophy of CA3 pyramidal neurons in response to chronic restraint stress, Neuroscience 81 (1997), pp. 689-697.

Gallagher and Pelleymounter, 1988 M. Gallagher and M.A. Pelleymounter, Spatial learning deficits in old rats: a model for memory decline I the aged, Neurobiol. Aging 9 (1988), pp. 549556.

Gao et al., 2002 H.B. Gao, M.H. Tong, Y.Q. Hu, Q.S. Guo, R. Ge and M.P. Hardy, Glucocorticoid induced apoptosis in rat Leydig cells, Endocrinology 143 (2002), pp. 130-148.

Gregus et al., 2005 A. Gregus, A.J. Wintink, A.C. Davis and L.E. Kalynchuk, Effect of repeated corticosterone injections and restraint stress on anxiety and depression-like behavior in male rats, Behav. Brain Res. 156 (2005), pp. 105-114.

Gresack and Frick, 2003 J.E. Gresack and K.M. Frick, Male mice exhibit better spatial working and reference memory than females in a water-escape radial arm maze task, Brain Res. 982 (2003) (1), pp. 98-107.

Hardy et al., 2002 M.P. Hardy, C.M. Sottas, R. Ge, C.R. McKittrick, K.L. Tamashiro, B.S. McEwen, S.G. Haider, C.M. Markham, R.J. Blanchard, D.C. Blanchard and R.R. Sakai, Trends of reproductive hormones in male rats during psychosocial stress: role of glucocorticoid metabolism in behavioral dominance, Biol. Reprod. 67 (2002), pp. 1750-1755.

Herman et al., 2001 J.P. Herman, B.R. Larson, D.B. Speert and A.F. Seasholtz, Hypothalamopituitary-adrenocortical dysregulation in aging F344/Brown-Norway F1 hybrid rats, Neurobiol. Aging 22 (2001) (2), pp. 323-332.

Hung et al., 2003 A.J. Hung, M.G. Stanbury, M. Shanabrough, T.L. Horvath, L.M. GarciaSegura and F. Naftolin, Estrogen, synaptic plasticity and hypothalamic reproductive aging, Exp. Gerontol. 38 (2003), pp. 53-59. 
Issa et al., 1990 A.M. Issa, W. Rowe, S. Gauthier and M.J. Meaney, Hypothalamic-pituitaryadrenal activity in aged, cognitively impaired and cognitively unimpaired rats, J. Neuro. 10 (1990), pp. 3247-5324.

Kitraki et al., 2004 E. Kitraki, O. Kremmyda, D. Youlatos, M.N. Alexis and C. Kittas, Genderdependent alterations in corticosteroid receptor status and spatial performance following 21 days of restraint stress, Neuroscience 125 (2004), pp. 47-55.

Lacreuse et al., 2005 A. Lacreuse, C.B. Kim, D.L. Rosene, R.J. Killiany, M.B. Moss, T.L. Moore, L. Chennareddi and J.G. Herndon, Sex, age, and training modulate spatial memory in the rhesus monkey (Macaca mulatta), Behav. Neurosci. 119 (2005), pp. 118-126.

LeFevre and McClintock, 1988 J. LeFevre and M.K. McClintock, Reproductive senescence in female rats: a longitudinal study of individual differences in estrous cycles and behavior, Biol. Reprod. 38 (1988) (4), pp. 780-789.

Li et al., 2004 C. Li, W.G. Brake, R.D. Romeo, J.C. Dunlop, M. Gordon, R. Buzescu, A.M. Magarinos, P.B. Allen, P. Greengard, V. Luine and B. McEwen, Estrogen alters hippocampal dendritic spine shape and enhances synaptic protein immunoreactivity and spatial memory in female mice, Proc. Natl. Acad. Sci. 101 (2004), pp. 2185-2190.

Luine and Hearns, 1990 V. Luine and M. Hearns, Spatial memory deficits in aged rats: contributions of the cholinergic system assessed by ChAT, Brain Res. 523 (1990), pp. 321-324.

Luine et al., 1990 V. Luine, D. Bowling and M. Hearns, Spatial memory deficits in aged rats: contributions of monoaminergic systems, Brain Res. 537 (1990), pp. 271-278.

Luine et al., 1994 V. Luine, C. Martinez, M. Villegas and B.S. McEwen, Repeated stress causes reversible impairments of spatial memory performance, Brain Res. 639 (1994), pp. 167-170.

Luine et al., 1996 V. Luine, C. Martinez, M. Villegas, A.M. Magarinos and B.S. McEwen, Restraint stress reversibly enhances spatial memory performance, Physiol. Behav. 59 (1996), pp. 27-32.

Luo et al., 2005 L. Luo, H. Chen and B.R. Zirkin, Temporal relationships among testosterone production, steroidogenic acute regulatory protein (StAR), and P450 side-chain cleavage enzyme (P450scc) during Leydig cell aging, J. Andrology 26 (2005), pp. 25-31.

Magarinos and McEwen, 1995 A.M. Magarinos and B.S. McEwen, Stress induced atrophy of apical dendrites of hippocampal CA3c neurons: comparison of stressors, Neuroscience 69 (1995), pp. 83-88.

Magiakou et al., 1997 M.A. Magiakou, G. Mastorakos, E. Webster and G.P. Chrousos, The hypothalamic-pituitary-adrenal axis and the female reproductive system, Ann. N. Y. Acad. Sci. 816 (1997), pp. 42-56. 
Markham et al., 2005 J.A. Markham, K.P. McKian, T.S. Stroup and J.M. Juraska, Sexually dimorphic aging of dendritic morphology in CA1 of hippocampus, Hippocampus 15 (2005) (1), pp. 97-103.

McEwen, 1999 B.S. McEwen, Stress and the aging hippocampus, Front. Neuroendocrinol. 20 (1999), pp. 49-70.

McEwen, 2001 B.S. McEwen, Plasticity of the hippocampus: adaptation to chronic stress and allostatic load, Ann. N. Y. Acad. Sci. 933 (2001), pp. 265-277. View Record in Scopus | Cited By in Scopus (290)

McEwen, 2002 B.S. McEwen, Sex, stress and the hippocampus: allostasis, allostatic load and the aging process, Neurobiol. Aging 23 (2002) (5), pp. 921-939.

McLaughlin et al., 2005 K.J. McLaughlin, S.E. Baran, R.L. Wright and C.D. Conrad, Chronic stress enhances spatial memory in ovariectomized female rats despite CA3 dendritic retraction: possible involvement of CA1 neurons, Neuroscience 135 (2005), pp. 1045-1054.

McLay et al., 1998 R.N. McLay, S.M. Freeman and J.E. Zadina, Chronic corticosterone impairs memory performance in the Barnes maze, Physiol. Behav. 63 (1998), pp. 933-937.

McNaughton et al., 1989 B.L. McNaughton, B. Leonard and L. Chen, Cortical-hippocampal interactions and cognitive mapping: a hypothesis based on reintegration of the parietal and interotemporal pathways for visual processing, Psychobiology 17 (1989), pp. 230-235.

Mizoguchi et al., 2000 K. Mizoguchi, M. Yuzurihara, A. Ishige, H. Sasaki, D.H. Chui and T. Tabira, Chronic stress induces impairment of spatial working memory because of prefrontal dopaminergic dysfunction, J. Neurosci. 20 (2000), pp. 1568-1574.

Monder et al., 1994 C. Monder, R.R. Sakai, Y. Miroff, D.C. Blanchard and R.J. Blanchard, Reciprocal change sin plasma corticosterone and testosterone in stressed male rats maintained in a visible burrow system: evidence for a mediating role of testicular 11 B-hydroxysteroid dehydrogenase, Endocrinology 134 (1994), pp. 1193-1198.

Mora et al., 1996 S. Mora, N. Dussaubat and G. Diaz-Veliz, Effects of the estrous cycle and ovarian hormones on behavioral indices of anxiety in female rats, Psychoneuroendocrinology 21 (1996), pp. 609-620.

Nacher et al., 2004 J. Nacher, K. Pham, V. Gil-Fernandez and B.S. McEwen, Chronic restraint stress and chronic corticosterone treatment modulate differentially the expression of molecules related to structural plasticity in the adult rat piriform cortex, Neuroscience 126 (2004), pp. 503509.

Nicholson et al., 2004 D.A. Nicholson, R. Yoshida, R.W. Berry, M. Gallagher and Y. Geinisman, Reduction in size of perforated postsynaptic densities in hippocampal axospinous synapses and age-related spatial learning impairments, J. Neurosci. 24 (2004), pp. 7649-7653. 
Nomikos and Spyraki, 1988 G.G. Nomikos and C. Spyraki, Influence of oestrogen on spontaneous and diazepam-induced exploration of rats in an elevated plus maze, Neuropharmacology 27 (1988), pp. 691-696.

O'Keefe and Dostrovsy, 1971 J.O. O'Keefe and J. Dostrovsy, The hippocampus as a spatial map. Preliminary evidence from unit activity in the freely-moving rats, Brain Res. 34 (1971), pp. 171-175.

Orr and Mann, 1992 T.E. Orr and D.R. Mann, Role of glucocorticoid in the stress-induced suppression of testicular steroidogenesis in adult male rats, Horm. Rehav. 26 (1992), pp. 350363.

Patchev et al., 1995 V.K. Patchev, S. Hayashi, C. Orikasa and O.F.X. Almeida, Implications of estrogen-dependent brain organization for gender differences in hypothalamo-pituitary-adrenal regulation, FASEB J. 9 (1995), pp. 419-423.

Perrot-Sinal et al., 1996 T.S. Perrot-Sinal, A.M. Kostenuik, K.P. Ossenkopp and M. Kavaliers, Sex differences in performance in the Morris water maze and the effects of initial nonstationary hidden platform training, Behav. Neurosci. 110 (1996), pp. 1309-1320.

Roof, 1993 R.L. Roof, Neonatal exogenous testosterone modifies sex difference in radial arm and Morris water maze performance in prepubescent and adult rats, Behav. Brain Res. 53 (1993), pp. 1-10.

Roof and Havens, 1992 R.L. Roof and M.D. Havens, Testosterone improves maze performance and induces development of a male hippocampus in females, Brain Res. 572 (1992) (1-2), pp. 310-313.

Sanders et al., 1982 B. Sanders, M.P. Soares and J.M. D'Aquila, The sex difference on one test of spatial visualization: a nontrivial difference, Child Dev. 53 (1982), pp. 1106-1110.

Sapolsky, 1999 R.M. Sapolsky, Glucocorticoids, stress, and their adverse neurological effects: relevance to aging, Exp. Gerontol. 34 (1999), pp. 721-732.

Scharfman et al., 2003 H.E. Scharfman, T.C. Mercurio, J.H. Goodman, M.A. Wilson and N.J. MacLusky, Hippocampal excitability increases during the estrous cycle in the rat: a potential role for brain-derived neurotrophic factor, J. Neurosci. 23 (2003), pp. 11641-11652.

Shors and Wood, 1995 T.J. Shors and G.E. Wood, Contribution of stress and gender to exploratory preferences for familiar versus unfamiliar conspecifics, Physiol. Behav. 58 (1995) (5), pp. 995-1002.

Shukitt-Hale et al., 2004 B. Shukitt-Hale, J.J. McEwen, A. Szprengiel and J.A. Joseph, Effect of age on the radial arm water maze-A test of spatial learning and memory, Neurobiology 25 (2004), pp. 223-229. 
Small et al., 2004 S.A. Small, M.K. Chawla, M. Buonocore, P.R. Rapp and C.A. Barnes, Imaging correlates of brain function in monkeys and rats isolates a hippocampal subregion differentially vulnerable to aging, Proc. Natl. Acad. Sci. 101 (2004) (18), pp. 7181-7186.

Steckler and Holsboer, 1999 T. Steckler and F. Holsboer, Corticotropin-releasing hormone receptor subtypes and emotion, Biol. Psychiatry 46 (1999), pp. 1480-1508.

Thomas et al., 2002 M.A. Thomas, H.B. Rice, D. Weinstock and R.L. Corwin, Effects of aging on food intake and body composition in rats, Physio. Behav. 76 (2002), pp. 487-500.

Veng et al., 2003 L.M. Veng, A.C. Granholm and G.M. Rose, Age-related sex differences in spatial learning and basal forebrain cholinergic neurons in Fischer 344 rats, Physiol. Behav. 80 (2003), pp. 27-36.

Williams et al., 1990 C.L. Williams, A.M. Barnett and W.H. Meck, Organizational effects of early gonadal secretions on sexual differentiation in spatial memory, Behav. Neurosci. 104 (1990), pp. 84-97.

Wilson et al., 2004 M. Wilson, P.R. Burghardt, K. Ford, M.B. Wilkinson and S.D. Primeaux, Anxiolytic effects of diazepam and ethanol in two behavioral models: comparison of males and females, Pharmacol. Biochem. Behav. 78 (2004) (3), pp. 445-458.

Wolf, 2003 O.T. Wolf, HPA axis and memory, Best Pract. Res., Clin. Endocrinol. Metab. 17 (2003), pp. 267-299. 